\title{
Effectiveness of temozolomide for primary glioblastoma multiforme in routine clinical practice
}

\author{
J. A. B. van Genugten $\cdot$ P. Leffers • \\ B. G. Baumert $\cdot$ H. Tjon-a-Fat $\cdot$ A. Twijnstra
}

Received: 1 April 2009/Accepted: 22 June 2009/Published online: 7 July 2009

(C) The Author(s) 2009. This article is published with open access at Springerlink.com

\begin{abstract}
Temozolomide has been used as a standard therapy for the treatment of newly diagnosed glioblastoma multiforme since 2005. To assess the effectiveness of temozolomide in routine clinical practice, we conducted an observational study at Maastricht University Medical Centre (MUMC). Data of patients receiving radiotherapy and temozolomide between January 2005 and January 2008 were retrieved from a clinical database (radiochemotherapy group), as were data of patients in a historical control group from the period before 2005 treated with radiotherapy only (radiotherapy group). The primary endpoint was overall survival. A total of 125 patients with GBM were selected to form the study cohort. Median survival benefit was 4 months: the median overall survival was 12 months (95\% CI, 9.7-14.3) in the group with radiochemotherapy with temozolomide, versus 8 months (95\% CI, 5.3-10.7) in the group with only radiotherapy. Progression-free survival was 7 months (95\% CI, 5.5-8.5) in the radiochemotherapy group and 4 months (95\% CI, 2.9-5.1) in the group with only radiotherapy. The two-year survival rate was $18 \%$
\end{abstract}

J. A. B. van Genugten · H. Tjon-a-Fat · A. Twijnstra ( $\square)$ Department of Neurology, Maastricht University Medical Centre, P.O. Box 5800, Maastricht 6202 AZ, The Netherlands

e-mail: jessie_van_genugten@hotmail.com

P. Leffers

Department of Epidemiology, Faculty of Health, Medicine and Life Sciences, University of Maastricht, P.O. Box 616, Maastricht 6200 MD, The Netherlands

\section{B. G. Baumert}

Department of Radiation Oncology (MAASTRO), GROW (Research Institute Growth and Development), Maastricht University Medical Centre, Maastricht, The Netherlands with radiochemotherapy with temozolomide against $4 \%$ with radiotherapy alone. Concomitant treatment with radiotherapy and temozolomide followed by adjuvant temozolomide resulted in grade III or IV haematological toxic effects in $9 \%$ of patients. The addition of temozolomide to radiotherapy in routine clinical practice for newly diagnosed glioblastoma resulted in a clinically meaningful survival benefit with minimal haematological toxicity, which confirms the experience of previous trials and justifies the continued use of temozolomide in routine clinical practice.

Keywords Temozolomide - Glioblastoma multiforme . Survival $\cdot$ Routine clinical practice $\cdot$ Radiotherapy

\section{Introduction}

Gliomas represent about half of all histologically verified primary CNS cancers among adults [1]. Glioblastoma multiforme (GBM), grade IV glioma, is the most frequent and most aggressive type of malignant brain tumour. The most characteristic features of GBM are necrosis and endothelial proliferation [2]. Despite the best available treatment, the clinical course is fatal, with a median survival of less than one year [3]. Factors that are prognostic for survival, apart from treatment modalities, are patient age, performance status and degree of surgical resection [1,3-13]. When a high grade glioma is suspected, maximal resection of the tumour is aimed for, on condition that neurological functions are spared. Such cytoreductive surgery is of limited value without adjuvant therapy. Until 2005, standard therapy consisted of surgical resection, followed by radiotherapy, which has been shown to prolong survival [14]. Unfortunately, the 
addition of various chemotherapeutics only resulted in marginal survival benefits $[15,16]$. Therefore, chemotherapy was not part of standard therapy for GBM in Europe and was only administered in the context of clinical trials. This changed after the publication of an EORTC/NCIC study in 2005, in which Stupp et al. demonstrated improved survival in patients with a histological diagnosis of glioblastoma multiforme, when concomitant temozolomide followed by adjuvant temozolomide was added to the standard treatment [17]. Similar results were seen in a smaller phase II trial by Athanassiou et al [18]. Temozolomide is an oral alkylating agent, with a lower toxicity profile than most other chemotherapeutic agents [19]. It can easily cross the blood-brain barrier, so that cerebrospinal fluid levels can reach at least $20 \%$ of those observed in the plasma [20]. Since the publication of these studies, temozolomide has been widely adopted as the new standard of care for patients with newly diagnosed GBM.

The outcome of randomised controlled trials cannot be directly applied without considering differences in patient characteristics between the trial population and patient populations in routine clinical practice. The objective of the present study was to assess the effectiveness of temozolomide in addition to radiotherapy in patients with newly diagnosed GBM in routine clinical practice. For this purpose, we conducted an observational study at Maastricht University Medical Centre (MUMC) among patients receiving radiotherapy and temozolomide and a historical control group of patients who were treated with radiotherapy only.

\section{Patients and methods}

\section{Study objectives}

The primary endpoint in this study was overall survival (OS), defined as the time from the starting date of radiotherapy to the date of death. Secondary endpoints were progression-free survival (PFS), 2-year overall survival and toxicity. PFS was defined from the starting date of radiotherapy to the date of tumour progression or death. The exact date of death was acquired from a database held at the General Registry Office of the Dept. of Radiation Oncology (MAASTRO Clinic). The safety and tolerability of temozolomide were estimated in the radiotherapy plus temozolomide group by assessing the haematological and non-haematological toxicity data from medical records and routine blood examinations. Although the National Cancer Institute has updated its Common Toxicity Criteria into version 3.0 (2006), we used version 2.0 to ensure better comparability with previous trials.
Eligibility

Since 1991, the Neuro-Oncology Department of the MUMC has been entering data on all neuro-oncological patients who are discussed in its weekly neuro-oncology meetings into a database. For the present study, we added data on patients who had not been discussed in these neurooncology meetings, but who had been registered in a pathology database (PALGA) with a histological GBM diagnosis. This allowed us to identify all patients aged 18 to 70 years who had a newly diagnosed and histologically confirmed supratentorial GBM according to the World Health Organization classification and who had been referred to the MUMC between January 2000 and January 2008. Data of patients who received radiotherapy and temozolomide between January 2005 and January 2008 were collected from this database (radiochemotherapy group). Data of patients receiving radiotherapy only were retrieved from the database for the period before 2005 (radiotherapy group). The subtypes of GBM, namely giant cell and gliosarcoma, were included.

Patients with a secondary glioblastoma, based on a prior histopathological diagnosis of a lower grade astrocytoma, were excluded. In cases where the first histopathological diagnosis was glioblastoma, but the clinical history was longer than one year, the pathologist was asked to do further immunohistochemical examinations to confirm or exclude the diagnosis of primary glioblastoma. Patients with gliomatosis cerebri were excluded, as well as patients with prior chemotherapy.

\section{Treatment}

All patients had surgery before radiotherapy. The extent of the surgery was defined as biopsy, partial resection or macroscopically complete resection and was determined from the surgical report and postoperative imaging, if performed. In the radiochemotherapy group, patients received radiotherapy with concomitant temozolomide, followed by adjuvant temozolomide, whereas the reference group received radiotherapy only (same radiotherapy treatment scheme with 30 fractions of 2 Gy to total dose of 60 Gy as used in the EORTC study by Stupp et al. [17]). Chemotherapy consisted of temozolomide at a dose of $75 \mathrm{mg} / \mathrm{m}^{2}$ per day, seven days a week for 42 consecutive days during radiotherapy. After a break of four weeks, patients received six cycles of adjuvant temozolomide at a dose of 150 to $200 \mathrm{mg} / \mathrm{m}^{2}$, on days 1 through 5 , every 28 days. Prophylactic co-trimoxazol against Pneumocystis carinii was given. Antiemetics, anticonvulsants and corticosteroids were prescribed if needed.

In case of disease progression, patients were assessed for their performance status and were offered 'salvage 
therapy', such as surgery, re-irradiation, second-line chemotherapy and/or supportive care.

Statistical analysis

Overall and progression-free survival curves were plotted using the method developed by Kaplan and Meier. The Cox proportional hazards model was used to adjust the hazard ratio for confounding and to improve the statistical efficiency. The prognostic factors tested were age, sex, WHO performance status and extent of surgery, since these have been reported in the literature as prognostic factors for survival outcome [1, 3-13].

We corrected for age per decade rather than the binary categories above or below 50 years, to obtain a more accurate measure of this variable. $P$-values lower than 0.05 were considered to be statistically significant. Statistical analysis was performed using the Statistical Package for the Social Sciences 15.0 (SPSS, Inc., Chicago, IL).

\section{Results}

\section{Patient characteristics}

One hundred and twenty-five patients with GBM were selected to form the study cohort. Patient details are outlined in Table 1. Median age was 56 years. Male/female ratio was 1.55 . The initial complaints were motor symptoms in $34 \%$ of the patients, headache in $45 \%$, epileptic seizures in $33 \%$, personality change in $12 \%$, and speech disorder in $26 \%$. The radiochemotherapy group consisted of 67 patients and the radiotherapy group of 58 patients. The median time from diagnosis to start of treatment was 5.9 weeks in the radiotherapy group and 3.9 weeks in the radiochemotherapy group. The baseline characteristics of the patients were not balanced as regards sex, extent of resection, corticosteroid use, giant cell or waiting time between diagnosis and the start of treatment.

\section{Treatment}

Median received radiation dose was 60 Gy (range, 4$60 \mathrm{~Gy})$. Ten patients ( $8 \%$ ) did not complete the entire radiotherapy, reasons including disease progression and death. Among the 67 patients who started radiochemotherapy, 93\% completed the concomitant phase with both radiotherapy and temozolomide. Of the 67 patients in the radiochemotherapy group, $75 \%$ started the adjuvant therapy with temozolomide after the 4-week break. Seventy percent of the radiochemotherapy group completed three cycles of temozolomide, and $40 \%$ had ultimately
Table 1 Patient characteristics

\begin{tabular}{|c|c|c|}
\hline Characteristic & $\begin{array}{l}\text { Radiotherapy } \\
(N=58)\end{array}$ & $\begin{array}{l}\text { Radiochemotherapy } \\
(N=67)\end{array}$ \\
\hline \multicolumn{3}{|l|}{ Age (year) } \\
\hline Median & 56 & 57 \\
\hline Range & $33-70$ & $25-70$ \\
\hline \multicolumn{3}{|l|}{ Sex-no. $(\%)$} \\
\hline Male & $29(50)$ & $47(70)$ \\
\hline Female & $29(50)$ & $20(30)$ \\
\hline \multicolumn{3}{|c|}{ WHO performance status, no. (\%) } \\
\hline 0 & $23(40)$ & $25(37)$ \\
\hline 1 & $24(41)$ & $30(45)$ \\
\hline 2 & $8(14)$ & $11(16)$ \\
\hline 3 & $3(5)$ & $1(2)$ \\
\hline \multicolumn{3}{|l|}{ Extent of surgery, no. (\%) } \\
\hline Biopsy & $33(57)$ & $30(45)$ \\
\hline Partial & $11(19)$ & $12(18)$ \\
\hline $\begin{array}{l}\text { Macroscopically } \\
\text { complete resection }\end{array}$ & $14(24)$ & $25(37)$ \\
\hline \multicolumn{3}{|c|}{ Time from diagnosis to treatment, wk } \\
\hline Median & 5.9 & 3.9 \\
\hline Range & $0.7-18$ & $2-8.4$ \\
\hline \multicolumn{3}{|c|}{ Corticosteroid therapy, no. (\%) } \\
\hline Yes & $39(67)$ & $46(69)$ \\
\hline No & $5(9)$ & $20(30)$ \\
\hline Data missing & $14(24)$ & $1(2)$ \\
\hline \multicolumn{3}{|c|}{ Subtype of glioblastoma, no. (\%) } \\
\hline Giant cell & $1(2)$ & $6(9)$ \\
\hline
\end{tabular}

Table 2 Temozolomide compliance

\begin{tabular}{lc}
\hline Treatment episode & \% Completed \\
\hline Week 3 RT concomitant & 100.0 \\
Week 5 RT concomitant & 97.0 \\
Total concomitant period & 92.5 \\
Cycle 1 & 74.6 \\
Cycle 2 & 73.1 \\
Cycle 3 & 70.1 \\
Cycle 4 & 44.7 \\
Cycle 5 & 43.2 \\
Cycle 6 & 40.3 \\
\hline
\end{tabular}

completed six cycles (see Table 2). The main reason for not completing concomitant and/or adjuvant temozolomide therapy in our study was disease progression, assessed by clinical deterioration and/or radiological assessment. Five patients $\mathbf{7 . 5 \%}$ ) in the radiochemotherapy group discontinued therapy because of haematological toxicity effects. 
Survival and progression

The follow-up ended on 8 July 2008. The unadjusted hazard ratio for death in the radiochemotherapy group compared to the radiotherapy group was 0.48 (95\% CI, $0.32-0.72 ; P<0.001)$. Multivariate analysis, including all considered covariates gave the same hazard ratio: 0.47 (95\% CI, 0.29-0.73; $P=0.001$ ). The Kaplan-Meier curve showed a median overall survival of 12 months $(95 \% \mathrm{CI}$, 5.3 to 10.7) in the radiochemotherapy group, versus 8 months (95\% CI, 9.7-14.3) in the group with radiotherapy only (Fig. 1 and Table 3). The estimated median survival benefit was therefore 4 months.

The unadjusted hazard ratio for PFS of radiochemotherapy compared to the radiotherapy was $0.58(95 \% \mathrm{CI}$, $0.39-0.85 ; P=0.006)$. The adjusted hazard ratio, corrected for all considered factors, was 0.59 (95\% CI, 0.38-

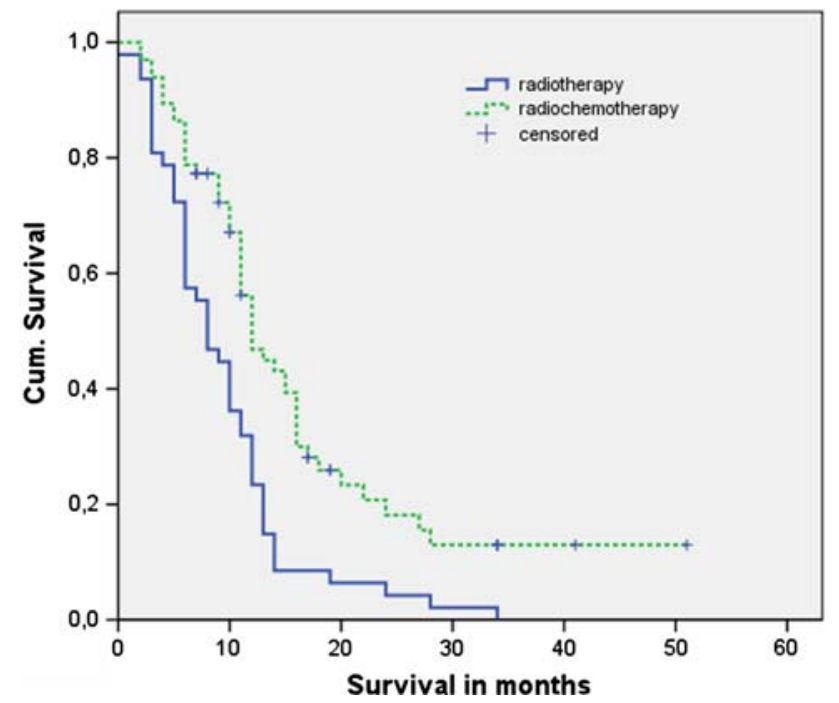

Fig. 1 Kaplan-Meier curves of overall survival: radiochemotherapy versus radiotherapy only. $P$ value $=0.000$. Censored: Still alive at end of study period $08-07-08$

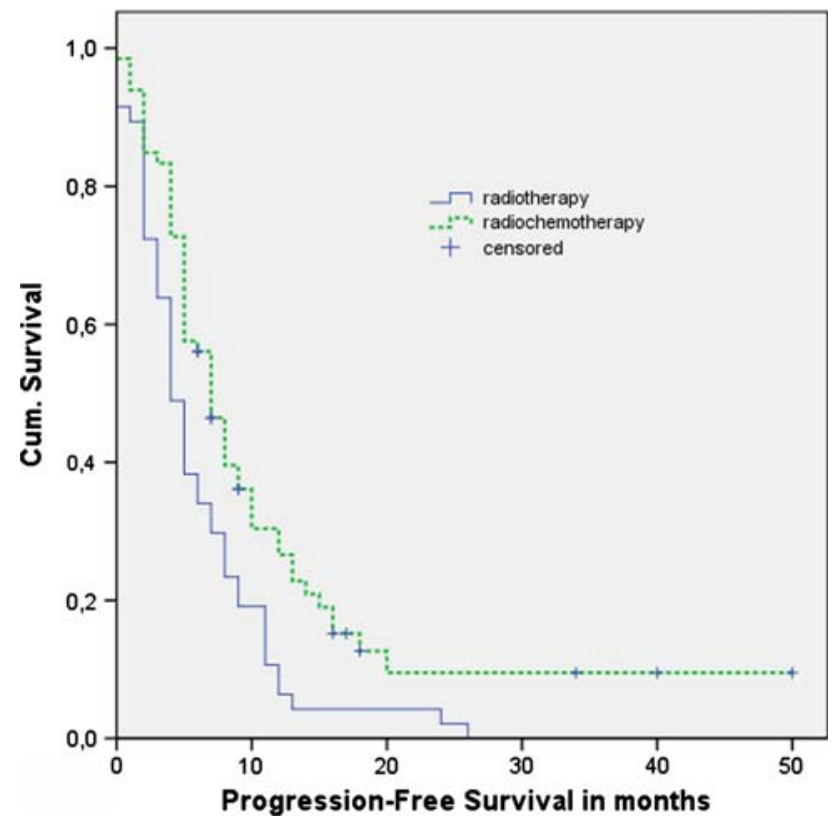

Fig. 2 Kaplan-Meier curves of progression-free survival: radiochemotherapy versus radiotherapy only. $P$-value $=0.003$. Censored: Still alive at end of study period 08-07-08

$0.91 ; P=0.016)$. The median progression-free survival was 7 months (95\% CI, 5.5-8.5) with radiochemotherapy and 4 months (95\% CI, 2.9-5.1) with only radiotherapy (Fig. 2, Table 3). The two-year survival rate was $18 \%$ in the radiochemotherapy group and $4 \%$ in the group with only radiotherapy.

The waiting time (medians 5.9 for radiotherapy and 3.9 weeks for radiochemotherapy) was also added to the Cox Regression model. The adjusted hazard ratio for death of radiotherapy plus temozolomide compared to radiotherapy alone, then became 0.42 (95\% CI, 0.24-0.73; $P=0.002)$. This change of the HR was caused by a clear positive association between waiting time and survival in
Table 3 Overall and progression-free survival

\begin{tabular}{lll}
\hline Variable & $\begin{array}{l}\text { Radiotherapy }(N=47) \\
\text { Value }(95 \% \mathrm{CI})\end{array}$ & $\begin{array}{l}\text { Radiochemotherapy }(N=66) \\
\text { Value }(95 \% \mathrm{CI})\end{array}$ \\
\hline $\begin{array}{l}\text { Median overall survival (months) } \\
\text { Overall survival (\%) }\end{array}$ & $8.0(5.3-10.7)$ & $12.0(9.7-14.3)$ \\
$\quad$ At 6 months & 57.4 & 78.8 \\
At 12 months & 23.4 & 46.8 \\
At 24 months & 4.3 & 18.2 \\
Median progression-free survival (months) & $4.0(2.9-5.1)$ & $7.0(5.5-8.5)$ \\
Progression-free survival (\%) & & \\
At 6 months & 34.0 & 56.1 \\
At 12 months & 6.4 & 26.6 \\
At 24 months & 2.1 & 9.5 \\
\hline
\end{tabular}


Fig. 3 Correlation between waiting time and survival in radiotherapy and radiochemotherapy groups radiotherapy

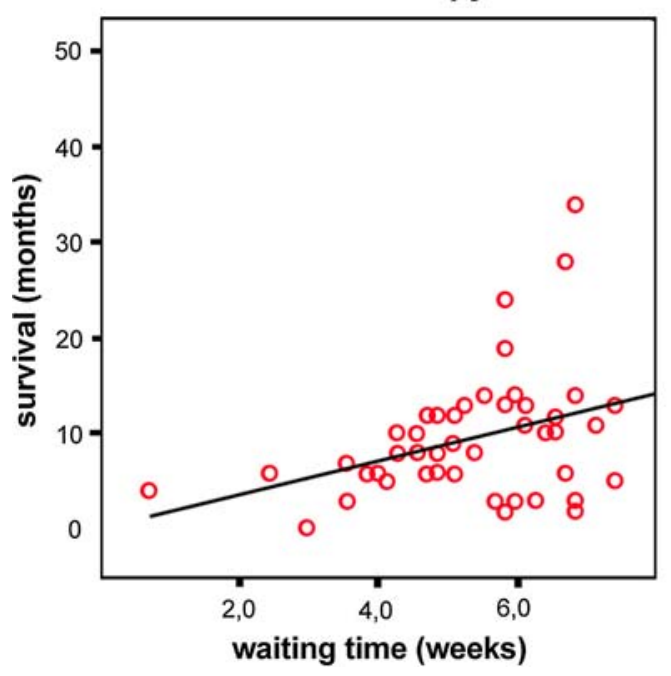

radiochemotherapy

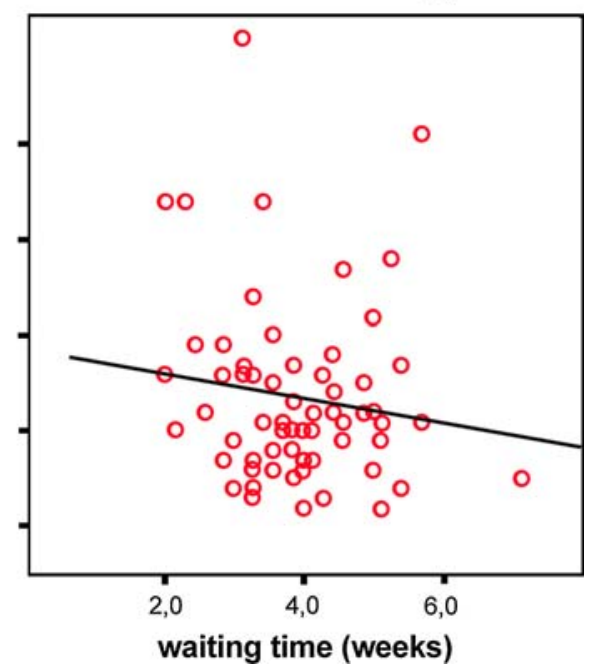

the radiotherapy group as is shown in Fig. 3. This association is probably due to patient selection. In the radiochemotherapy period (2005-2008), there was a weak inverse association.

\section{Subgroup analysis}

Survival was analysed according to the prognostic factors. Radiochemotherapy was associated with a significant improvement in median overall survival in nearly all subgroups of patients, with the exception of the relatively large subgroup of patients who underwent biopsy only and the patients with a WHO performance status 1 (whereas those with the poorer performance status WHO 2 and 3 did show a significant improvement in median survival).

\section{Comparison with EORTC/NCIC trial}

To achieve the best possible comparison of the effect of this new treatment in routine clinical practice and in the EORTC/NCIC trial, we calculated what the effect of the treatment in our setting would have been for the patients of the EORTC/NCIC trial. To this end, we entered the mean patient characteristics of their trial population (Table 4) into our Cox regression model. The survival curve in Fig. 4 shows the effect of treatment on the survival of an average patient in the EORTC trial. This patient would be expected to survive for 10.0 months with radiotherapy and 14.2 months when temozolomide was added to the treatment. An average patient in our routine clinical practice cohort survived 9.0 months with radiotherapy and 12.9 months with radiochemotherapy (Fig. 5).
Toxicity

Detailed toxicity data were not recorded for the radiotherapy group in the neurology treatment charts, whereas temozolomide-related haematotoxicity was recorded for each patient in the radiochemotherapy group. Six patients $(9 \%)$ had any type of grade III or IV haematological toxic effect. In five of these cases chemotherapy was discontinued. Anti-epileptic drugs were given to $41 \%$ of patients in the radiotherapy group and $47 \%$ of patients in the radiochemotherapy group. Only 5\% received the non-enzyme inducing valproic acid.

Treatment after disease progression

At the moment of disease progression, four patients in both treatment groups underwent a second surgery, seven patients were re-irradiated, 16 patients received additional cycles of temozolomide and four patients received secondline chemotherapy other than temozolomide. In total, $17 \%$ of patients in the radiochemotherapy group and $18 \%$ of patients in the radiotherapy group received salvage chemotherapy (see Table 5). Salvage chemotherapy consisted of additional cycles of temozolomide in $67 \%$ of patients in the radiochemotherapy group and $100 \%$ of patients in the radiotherapy group.

\section{Discussion}

Since the publication of randomised trials in which patients with newly diagnosed glioblastoma multiforme (GBM) showed a prolonged survival when treated with both radiotherapy and temozolomide, this chemotherapeutic 
Table 4 Patient

characteristics-comparison

with EORTC/NCIC trial

\begin{tabular}{llll}
\hline Characteristic & $\frac{\text { Present study }}{\text { RT }}$ & Stupp et al. \\
& RT + TMZ & RT + TMZ
\end{tabular}

Age (year)

Median

Sex $(\%)$

Male

Female

WHO performance status (\%)

0

1

2

3

Extent of surgery (\%)

Biopsy

Partial

Macroscopically complete resection

Time from diagnosis to treatment-wk

Median

56

57

57

56

$50 \quad 70$

$50 \quad 30$

64

$40 \quad 37$

$41 \quad 45$

$14 \quad 16$

5

2

$57 \quad 45$

\section{5}

18

37

5.9

3.9

5.0

5.0

Corticosteroid therapy $(\%)$

Yes

No

Data missing

9

24

Subtype of glioblastoma (\%)

Giant cell

2

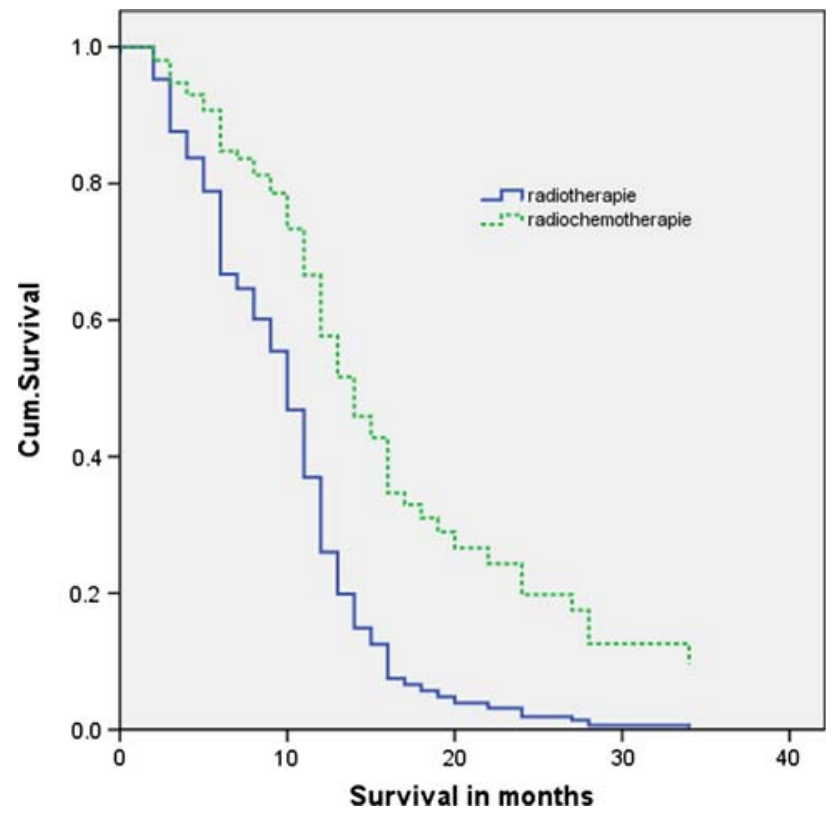

Fig. 4 Survival curve of an average patient of the EORTC/NCIC trial

agent has been implemented as the standard therapy. To verify the value of this established treatment with temozolomide in routine clinical practice, we conducted an observational study at the Maastricht University Medical

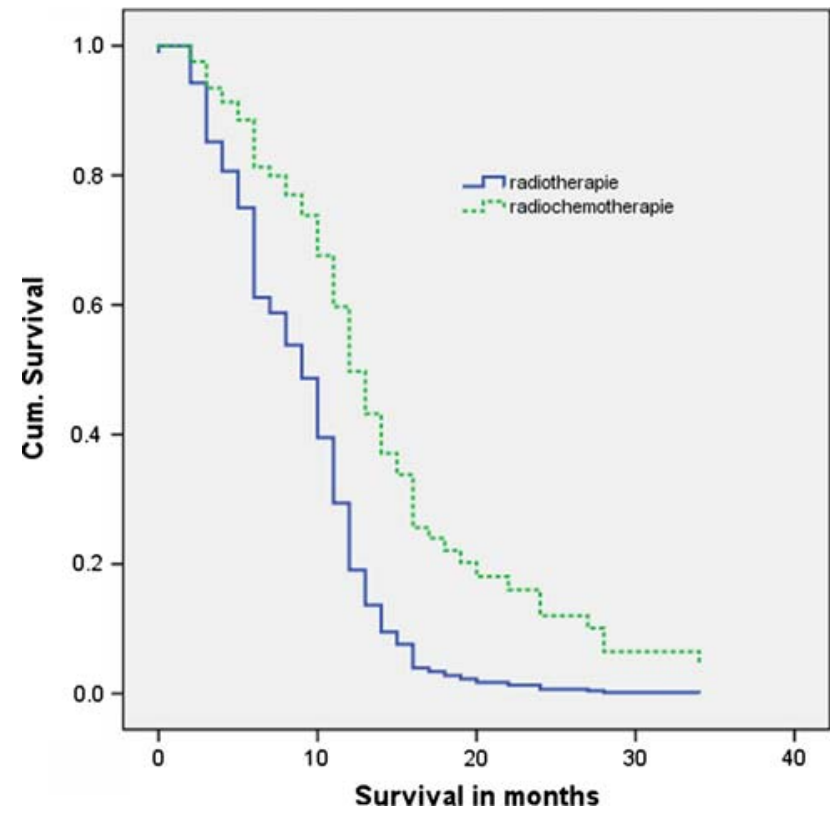

Fig. 5 Survival curve of an average patient of selected patients from clinical practice

Centre, comparing survival in a cohort of patients receiving radiochemotherapy with that in a historical control group receiving radiotherapy only. Our study showed that radiochemotherapy is indeed also effective in routine clinical 
Table 5 Salvage treatment

\begin{tabular}{llc}
\hline Type of treatment & $\begin{array}{l}\text { Radiotherapy } \\
(N=47)\end{array}$ & $\begin{array}{l}\text { Radiochemotherapy } \\
(N=66)\end{array}$ \\
\hline Second surgery, no. $(\%)$ & $0(0)$ & $4(6)$ \\
Re-irradiation, no. $(\%)$ & $1(2)$ & $6(9)$ \\
Chemotherapy, no. $(\%)$ & $8(17)$ & $12(18)$ \\
Temozolomide & $8(17)$ & $8(12)$ \\
Other than temozolomide & $0(0)$ & $4(6)$ \\
\hline
\end{tabular}

practice, although the effectiveness is somewhat different from that in the published trials.

We found a median overall survival of 12.0 months in the group with radiochemotherapy and 8.0 months in the group with radiotherapy only. The median survival benefit in the present study was thus 4 months, which is lower than the 5.7 months benefit found by Athanassiou et al. but higher than the 2.5 months median survival benefit found by Stupp et al. (Table 6). [17, 18] In the study by Athanassiou et al. the dose of temozolomide during the concomitant phase was the same, but temozolomide was given every two instead of four weeks during the adjuvant phase. It remains unclear whether that is an explanation for the higher survival benefit in their study.

In our study, $40 \%$ of all patients in the radiochemotherapy group completed the concomitant and total adjuvant treatments, which was slightly higher than the $36.6 \%$ in the study by Stupp et al. while $61.4 \%$ of the patients in the trial by Athanassiou et al. completed six cycles of temozolomide. The difference in compliance between the present study and the phase III trial by Stupp et al. can only partially explain the larger median survival benefit in our study.

The decrease in waiting time with median times of 5.9 until 2005 and 3.9 in recent years is the consequence of a more time-efficient approach to patients with GBM introduced in recent years. It has been reported that a delay until

Table 6 Overall and progression-free survival-comparison with trials

\begin{tabular}{|c|c|c|}
\hline Variable & $\begin{array}{l}\text { Radiotherapy } \\
\text { Median }(95 \% \text { CI) }\end{array}$ & $\begin{array}{l}\text { Radiochemotherapy } \\
\text { Median }(95 \% \text { CI) }\end{array}$ \\
\hline \multicolumn{3}{|c|}{ Overall survival (months) } \\
\hline Present study & $8.0(5.5-10.5)$ & $12.0(9.7-14.3)$ \\
\hline Stupp et al. & $12.1(11.2-13.0)$ & $14.6(13.2-16.8)$ \\
\hline Athanassiou et al. & $7.7(5.3-9.2)$ & $13.4(9.6-17.1)$ \\
\hline \multicolumn{3}{|c|}{ Progression-free survival (months) } \\
\hline Present study & $4.0(2.9-5.1)$ & $7.0(5.5-8.5)$ \\
\hline Stupp et al. & $5.0(4.2-5.5)$ & $6.9(5.8-8.2)$ \\
\hline Athanassiou et al. & $5.2(4.0-7.4)$ & $10.8(8.1-14.7)$ \\
\hline
\end{tabular}

the first fraction of radiotherapy after surgery or after first consultation in the radiotherapy department results in a clinically significant reduction of survival for patients with glioblastoma [21-24]. We investigated the correlation between waiting time and survival and found a positive association in the radiotherapy group, which seems to be responsible for the stronger therapeutic effect (lower hazard ratio) after correction for waiting time. This association is probably caused by selection: patients assessed as 'better' in terms of performance status had to wait longer for treatment. Recently Blumenthal et al. also showed that delaying initiation of radiotherapy did not show reduction in survival, but a superior outcome instead [25]. These comparable results may be based on the same kind of patient selection for early treatment as was mentioned before. The difference in waiting time between the two cohorts could not explain the larger survival benefit in the present study compared to the EORTC/NCIC trial (median waiting time being 5 weeks in both groups).

The fact that the median overall survival was shorter in both groups in the present study than in the EORTC/NCIC trial can at least partly be explained by a larger number of patients being surgically treated with biopsy only. In our study, $57 \%$ and $45 \%$ of the radiotherapy and radiochemotherapy groups, respectively, had a biopsy only, versus $16 \%$ and $17 \%$ in the EORTC/NCIC trial. Most studies have shown a significant survival advantage for patients undergoing a more extensive degree of resection $[3,5,10,12$, 13]. Also one has to keep in mind that both our study and EORTC/NCIC trial did not show a clear survival advantage in the subgroup with patients undergoing biopsy [17].

Although we have corrected for the possible confounding factors, the slightly worse results in our MUMC cohort can also be the consequence of the well-known phenomenon of selection bias from patient selection for clinical trials.

Since a primary goal of therapy for patients with GBM is improvement or maintenance of quality of life, delaying disease progression by extending the progression-free survival is very important. The present study demonstrated that the addition of temozolomide to radiotherapy achieved a prolongation of the median progression-free survival from 4 months in the radiotherapy group to 7 months in the radiochemotherapy group (Table 6).

Survival from progression, that is, the difference between OS and PFS, was shorter in our study (4.0 and 5.0 months in the radiotherapy and radiochemotherapy groups, respectively) than in the EORTC/NCIC trial (7.1 and 7.7 months). This may be caused by less salvage treatment. In our study, only $17 \%$ of the radiotherapy group and $18 \%$ of the radiochemotherapy group received salvage chemotherapy, whereas in the EORTC/NCIC trial, $72 \%$ and $58 \%$, respectively, received salvage chemotherapy after 
progression. In the EORTC/NCIC trial, $23 \%$ of patients in both treatment groups underwent repeat surgery, compared to only $4 \%$ in our study. Re-irradiation was given to $6 \%$ of patients in the present study (3\% before 2005 and $9 \%$ since 2005). The number of patients receiving re-irradiation in the EORTC/NCIC trial was not reported [17].

Apart from biopsy and waiting time, reported patient characteristics of the randomised EORTC trial and our study were quite similar (Table 4). By estimating the treatment effect of the EORTC/NCIC trial population in our setting from our Cox regression model (Fig. 4), we showed that a quite similar survival benefit would have been accomplished as what was achieved in our study population (4.2 instead of 3.9 months) and a higher survival of 10 months with radiotherapy and 14.2 months with radiochemotherapy.

Among the 125 cases of glioblastoma multiforme included in this study, only seven patients $(5.6 \%)$ had giant cell glioblastoma multiforme. Normally, giant cell GBM (GC-GBM) constitutes approximately $5 \%$ of the cases of glioblastoma multiforme, and GC-GBM is recognised as a distinct clinicopathologic entity in the WHO 2007 classification $[2,26]$. The difference in the prevalence of the giant cell subtype between the two groups in our study (Table 1) is remarkable: one patient in the radiotherapy group and six in the radiochemotherapy group. This might be a coincidence but it is more probable due to a change in pathological analysis. Patients with giant cell GBM were on average 16.5 years younger than those with non giant cell GBM and macroscopically complete resection was possible in $71 \%$ patients versus $29 \%$ in non giant cell, probably contributing to a better survival [27]. Unfortunately, the Stupp trial did not mention the number of patients with giant cell subtype.

Our results demonstrate that temozolomide combined with radiotherapy is an effective chemotherapeutic agent for patients with primary glioblastoma multiforme in clinical practice.

Open Access This article is distributed under the terms of the Creative Commons Attribution Noncommercial License which permits any noncommercial use, distribution, and reproduction in any medium, provided the original author(s) and source are credited.

\section{References}

1. Ohgaki H, Kleihues P (2005) Epidemiology and etiology of gliomas. Acta Neuropathol 109(1):93-108

2. Louis DN, Ohgaki H, Wiestler OD, Cavenee WK, Burger PC, Jouvet A et al (2007) The 2007 WHO classification of tumours of the central nervous system. Acta Neuropathol 114(2):97-109

3. Laws ER, Parney IF, Huang W, Anderson F, Morris AM, Asher A et al (2003) Survival following surgery and prognostic factors for recently diagnosed malignant glioma: data from the glioma outcomes project. J Neurosurg 99(3):467-473 see comment
4. Curran WJ Jr, Scott CB, Horton J, Nelson JS, Weinstein AS, Fischbach AJ et al (1993) Recursive partitioning analysis of prognostic factors in three Radiation Therapy Oncology Group malignant glioma trials. J Natl Cancer Inst 85(9):704-710 see comment

5. Lacroix M, Abi-Said D, Fourney DR, Gokaslan ZL, Shi W, DeMonte $\mathrm{F}$ et al (2001) A multivariate analysis of 416 patients with glioblastoma multiforme: prognosis, extent of resection, and survival. J Neurosurg 95(2):190-198 see comment

6. Kreth FW, Warnke PC, Scheremet R, Ostertag CB (1993) Surgical resection and radiation therapy versus biopsy and radiation therapy in the treatment of glioblastoma multiforme. J Neurosurg 78(5):762-766

7. Chang CH, Horton J, Schoenfeld D, Salazer O, Perez-Tamayo R, Kramer S et al (1983) Comparison of postoperative radiotherapy and combined postoperative radiotherapy and chemotherapy in the multidisciplinary management of malignant gliomas. A joint Radiation Therapy Oncology Group and Eastern Cooperative Oncology Group study. Cancer 52(6):997-1007 Sep 15

8. Sandberg-Wollheim M, Malmstrom P, Stromblad LG, Anderson H, Borgstrom S, Brun A et al (1991) A randomized study of chemotherapy with procarbazine, vincristine, and lomustine with and without radiation therapy for astrocytoma grades 3 and/or 4 . Cancer 68(1):22-29

9. Slotman BJ, Kralendonk JH, van Alphen HA, Kamphorst W, Karim AB (1996) Hypofractionated radiation therapy in patients with glioblastoma multiforme: results of treatment and impact of prognostic factors. Int J Radiat Oncol Biol Phys 34(4):895-898

10. Devaux BC, O'Fallon JR, Kelly PJ (1993) Resection, biopsy, and survival in malignant glial neoplasms. A retrospective study of clinical parameters, therapy, and outcome. J Neurosurg 78(5): 767-775

11. Shapiro WR, Green SB, Burger PC, Mahaley MS Jr, Selker RG, VanGilder JC et al (1989) Randomized trial of three chemotherapy regimens, two radiotherapy regimens, two radiotherapy regimens in postoperative treatment of malignant glioma Brain Tumor Cooperative Group Trial 8001. J Neurosurg 71(1):1-9

12. Stummer W, Reulen HJ, Meinel T, Pichlmeider U, Schumacher W, Tonn JC et al (2008) Extent of resection and survival in glioblastoma multiforme: identification of and adjustment for bias. Neurosurgery 62(3):564-576

13. Gorlia T, van den Bent MJ, Hegi ME, Mirimanoff RO, Weller M, Cairncross JG et al (2008) Nomograms for predicting survival of patients with newly diagnosed glioblastoma: prognostic factor analysis of EORTC and NCIC trial 26981-22981/CE.3. Lancet Oncol 9(1):29-38 see comment

14. Walker MD, Green SB, Byar DP, Alexander E Jr, Batzdorf U, Brooks WH et al (1980) Randomized comparisons of radiotherapy and nitrosoureas for the treatment of malignant glioma after surgery. N Engl J Med 303(23):1323-1329

15. Fine HA, Dear KB, Loeffler JS, Black PM, Canellos GP (1993) Meta-analysis of radiation therapy with and without adjuvant chemotherapy for malignant gliomas in adults. Cancer 71(8): 2585-2597 see comment

16. Stewart LA (2002) Chemotherapy in adult high-grade glioma: a systematic review and meta-analysis of individual patient data from 12 randomised trials. Lancet 359(9311):1011-1018

17. Stupp R, Mason WP, van den Bent MJ, Weller M, Fisher B, Taphoorn MJ et al (2005) Radiotherapy plus concomitant and adjuvant temozolomide for glioblastoma. N Engl J Med 352(10): 987-996 see comment

18. Athanassiou H, Synodinou M, Maragoudakis E, Paraskevaidis M, Verigos C, Misailidou D et al (2005) Randomized phase II study of temozolomide and radiotherapy compared with radiotherapy alone in newly diagnosed glioblastoma multiforme. J Clin Oncol 23(10):2372-2377 Apr 1 
19. Yung WK, Albright RE, Olson J, Fredericks R, Fink K, Prados MD et al (2000) A phase II study of temozolomide vs. procarbazine in patients with glioblastoma multiforme at first relapse. Br J Cancer 83(5):588-593

20. Ostermann S, Csajka C, Buclin T, Leyvraz S, Lejeune F, Decosterd LA et al (2004) Plasma and cerebrospinal fluid population pharmacokinetics of temozolomide in malignant glioma patients. Clin Cancer Res 10(11):3728-3736 Jun 1

21. Irwin C, Hunn M, Purdie G, Hamilton D (2007) Delay in radiotherapy shortens survival in patients with high grade glioma. J Neurooncol 85(3):339-343

22. Do V, Gebski V, Barton MB (2000) The effect of waiting for radiotherapy for grade III/IV gliomas. Radiother Oncol 57(2): 131-136 see comment

23. Burnet NG, Jena R, Jefferies SJ, Stenning SP, Kirkby NF (2006) Mathematical modelling of survival of glioblastoma patients suggests a role for radiotherapy dose escalation and predicts poorer outcome after delay to start treatment. Clin Oncol (R Coll Radiol) 18(2):93-103 see comment

24. Lutterbach J (2003) Live fast, die early? The deleterious effects of waiting time in patients with glioblastoma. Anticancer Drugs 14(6):447-448

25. Blumenthal DT, Won M, Mehta MP, Curran WJ, Souhami L, Michalski JM et al (2009) Short delay in initiation of radiotherapy may not affect outcome of patients with glioblastoma: a secondary analysis from the radiation therapy oncology group database. J Clin Oncol 27(5):733-739

26. Miller CR, Perry A (2007) Glioblastoma. Arch Pathol Lab Med 131(3):397-406

27. Margetts JC, Kalyan-Raman UP (1989) Giant-celled glioblastoma of brain. A clinico-pathological and radiological study of ten cases (including immunohistochemistry and ultrastructure). Cancer 63(3):524-531 